Meta

Journal des traducteurs

Translators' Journal

\title{
Index du volume 29
}

Volume 29, numéro 4, décembre 1984

URI : https://id.erudit.org/iderudit/003178ar

DOI : https://doi.org/10.7202/003178ar

Aller au sommaire du numéro

Éditeur(s)

Les Presses de l'Université de Montréal

ISSN

0026-0452 (imprimé)

1492-1421 (numérique)

Découvrir la revue

Citer ce document

(1984). Index du volume 29. Meta, 29(4), 426-441.

https://doi.org/10.7202/003178ar

Ce document est protégé par la loi sur le droit d'auteur. L'utilisation des services d'Érudit (y compris la reproduction) est assujettie à sa politique d'utilisation que vous pouvez consulter en ligne.

https://apropos.erudit.org/fr/usagers/politique-dutilisation/ 


\section{Index \\ du \\ volume 29}

\section{A. ARTICLES}

Bagge, Ch. : Traduction et création, $n^{\circ} 2$, p. 135.

Baker, W. J. : An Integrated Perspective on Cognitive Strategies in Language Processing, $n^{\circ} 1$, p. 81.

Bouton, Ch. P. : Le cerveau du traducteur. De quelques propositions sur ce thème, no 1, p. 44 .

Branchereau, L. : La zone du langage et l'aphasie : enseignement standard et cas particulier, $\mathrm{n}^{\circ} 1, \mathrm{p} .10$

Brisset, A. : Poésie : le sens en effet. Étude d'un translème, $n^{\circ} 3$, p. 259.

Clas, A. : Éditorial, $n^{\circ} 4$, p. 339.

Clercq, M. de : Le problème de l'intertextualité dans la traduction littéraire : l'exemple de Beckett, $\mathrm{n}^{\circ} 3$, p. 253.

Deschamps, R. : L'agestion ou la gestion en temps de crise, $n^{\circ} 4$, p. 364.

Di Virgilio, P. S. : The Sense of a Beginning : The Dynamics of Context in Translation, $n^{\circ} 2, p .115$.

Fillion, L. R. : Gestion et traduction. Un mariage de raison, $\mathbf{n}^{\circ} 4$, p. 340.

Folkart, B. : A Thing-bound Approach to the Practice and Teaching of Technical Translation, no 3, p. 229.

Frenette, R. : Le cabinet de traduction : une gestion de l'évolution, $n^{\circ} 4$, p. 352.

Gouadec, D. : Les micro-banques de terminologie - Instruments d'une pratique ; outils d'apprentissage Le système "TERM.X ", n 4, p. 367.

Henry, R. : Points for Inquiry into Total Translation. A Review of J. C. Catford's, A Linguistic Theory of Translation, $\mathrm{n}^{\circ} 2, \mathrm{p} .152$.

Jarema, G. : Les problèmes de la terminologie aphasiologique, no 1, p. 99.

Joanette, Y. : La zone du langage et l'aphasie : enseignement standard et cas particulier, $\mathrm{n}^{\circ} 1$, p. 10.

Joanette, $Y$. : Cerveau et langages : nouvelles perspectives, $n^{\circ} 1, p .27$.

Kachroo, B. : Textual Cohesion and Translation, $n^{\circ} 2$, p. 128.

Larose, R. : Le rôle des annotations de textes dans l'enseignement de la traduction, no 2 , p. 143.

Lebrun A. R. : La neurophysiologie du bilinguisme, $n^{\circ} 1$, p. 36.

Lecours, A. R. : La zone du langage et l'aphasie : enseignement standard et cas particulier, $n^{\circ} 1$, p. 10.

Lecours, A. R. : Cerveau et langages : nouvelles perspectives, $n^{\circ} 1$, p. 27.

Le Dorze, G. : Processus de lexicalisation. Modèles psychologiques et leur application à l'étude de l'aphasie et de la traduction, $\mathrm{n}^{\circ} 1$, p. 68 .

Le Meur, A. : Les micro-banques de terminologie - Instruments d'une pratique ; outils d'apprentissage Le système "TERM.X", n 4, p. 367.

MacNamee, T. : La terminologie de la neurolinguistique : perspectives diachroniques, $n^{0} 1$, p. 91.

Murith, P. : Service de traduction d'une entreprise d'assurances de taille moyenne. Le traducteur allemand/ français, $n^{\circ} 4$, p. 362.

Nespoulous, J.-L. : En guise d'introduction... Neurolinguistique, psycholinguistique et traduction, $n^{\circ} 1$, p. 5.

Nespoulous, J.-L. : Processus de lexicalisation. Modèles psychologiques et leur application à l'étude de l'aphasie et de la traduction, $n^{\circ} 1$, p. 68.

Paradis, M. : Aphasie et traduction, $n^{\circ} 1$, p. 57.

Prideaux, G. : An Integrated Perspective on Cognitive Strategies in Language Processing, $\mathbf{n}^{\mathrm{0}} 1$, p. 81.

Romney, Cl. : Problèmes culturels de la traduction d'Alice in Wonderland en français, $\mathrm{n}^{\circ} 3$, p. 267.

Schneider, T. : Problems Incorporated. Translation Management in an Industrial Environment, nº 4, p. 359.

Velan, Y. : L'exemple de Plaute, nº 3, p. 247. 


\section{B. ÉTUDES TERMINOLOGIQUES ET LINGUISTIQUES}

Anderson, L. : Courrier des lecteurs, $n^{\circ} 3$, p. 312.

Bélanger, G. : Les hydrocarbures, $n^{\circ} 4$, p. 387.

Brisebois, M. : Marginal, $\mathrm{n}^{\circ}$ 3, p. 307.

Chansou, M. : Calques et créations linguistiques, $\mathrm{n}^{\circ} 3$, p. 281.

Cohen, B. : Un nouveau type de dictionnaire utile aux traducteurs : le DEC du français moderne, $\mathrm{n}^{\circ} 2$, p. 159.

Comité de terminologie française, Ordre des comptables agréés du Québec : Société - association compagnie - corporation, $\mathrm{n}^{\circ} 3$, p. 297.

Elnitsky, L. : Un nouveau type de dictionnaire utile aux traducteurs : le DEC du français moderne, $n^{\circ} 2$, p. 159.

Flamin, B. : On nous écrit de ..., nº 2, p. 196.

Gile, D. : La recherche terminologique dans la traduction scientifique et technique japonais-français : une synthèse, $\mathrm{n}^{\circ} 3$, p. 285.

Gile, D. : On nous écrit de..., no 4, p. 404.

Haché, J. : Marginal, $\mathrm{n}^{\circ}$ 3, p. 307.

Kennedy, E. : In Statistics, Errors aren’t Errors?, nº 4, p. 375.

Loffler-Laurian, A.-M. : Norme de communication et traduction de textes techniques, $\mathrm{n}^{\circ} 2, \mathrm{p} .175$.

Pelletier, C. : Les télécommunications modernes dans l'entreprise, $\mathrm{n}^{\circ} 2$, p. 189.

Proulx, M. : Etude terminologique des scies manuelles, $n^{\circ} 2$, p. $182 ; n^{\circ} 3$, p. $291 ; n^{\circ} 4$, p. 395.

Rohaert, A. : Parlement européen, $n^{\circ} 2$, p. 198.

Sournia, J.-C. : On nous écrit de...., no 4, p. 404.

Stellbrink, H.-J. : On nous écrit de..., no 2, p. 196.

\section{DOCUMENTATION}

Serré, R. : Ouvrages récents qui englobent un lexique ou un glossaire, no 2, p. $201 ; n^{\circ} 3$, p. $315 ; n^{\circ} 4$, p. 405. Spilka, I. V. : Bibliographie, $n^{\circ} 2$, p. $200 ; n^{\circ} 3$, p. $315 ; n^{\circ} 4$, p. 405.

\section{COMPTES RENDUS}

Baudot, J. : Informatique, $\mathrm{n}^{\circ} 2$, p. 208.

Béjoint, H. : Lexicography in the Electronic Age, no 4, p. 415

Breton, F. : Dictionnaire de l'eau, no 4, p. 407.

Brisebois-Mathieu, M. : Règles de catalogage anglo-américaines, $n^{0} 3$, p. 321 .

Darbelnet, J. : Dictionnaire des termes nouveaux des sciences et des techniques, n 3, p. 320.

De Chezet, J.-P. : Écrire et traduire, nº 4. p. 401.

Elnitsky, L. : Lexique russe-français de mécanique des sols, $n^{\circ} 4$, p. 410.

Flamand, J. : Guide du rédacteur de l'administration fédérale, nº 2 , p. 202.

Gémar, J.-C. : Terminologie de l'avant-projet de convention relatif à un droit européen des marques, $n^{\circ} 3$, p. 323.

Gémar, J.-C. : Terminologie du brevet européen, no 3, p. 324.

Gémar, J.-C. : Terminologie de la société anonyme européenne, no 3 , p. 325.

Gémar, J.C. : Rédaction des lois, $n^{\circ} 4$, p. 417.

Michon, H. : Dictionnaire de l'eau, $n^{\circ} 4$, p. 407.

Paré, M. : Dictionnaire des anglicismes, no 2, p. 206.

Portelance, Ch. : Le franglais vingt ans après, $n^{\circ} 4$, p. 416. 
Sénécal, A. : La langue française de la science et de la technique, $\mathrm{n}^{\circ} 2$, p. 209

Serré, R. : Dictionnaire contextuel anglais-français de la corrosion, $\mathrm{n}^{\circ} 3$, p. 316 .

Slote, D. : The Other Shakespeare, no 3 , p. 326.

Slote, D. : I, Lars Hard, no 3 , p. 326.

Spilka, I. V. : Richesses et particularités de la langue écrite au Québec, $\mathrm{n}^{\circ} 2$, p. 211 .

Spilka, I. V. : Comprendre pour traduire, perfectionnement linguistique en français, nº 3, p. 323.

\section{E. BLOC NOTES}

Baudot, J. : Développement des langues africaines : de Yaoundé à Zanzibar, n 2, p. 212.

Baudot, J. : EACROTANAL : Séminaire de terminologie, Zanzibar, nº 3, p. 333.

Bon, L. B. : Je, tu, il ou elle, nous, vous, ils ou elles, $n^{\circ} 2$, p. 218.

Chénard-Nantel, C. : Terminologie et communication, $\mathrm{n}^{\mathrm{0}} 2$ 2, p. 215.

Clas, A. : Développement des langues africaines : de Yaoundé à Zanzibar, no 2, p. 212.

Clas, A. : EACROTANAL : Séminaire de terminologie, Zanzibar, $\mathrm{n}^{\circ} 3$, p. 333.

Coughlin, J. : Should Court Interpreters and Conference Interpreters Be Trained in Separate Academic Programs ?, no 4, p. 420

Flamand, J. : Qu'est-ce qu'une bonne traduction?, no 3 , p. 330.

Griffith, L. : Nit Orchard, Pickers Wanted, no 3, p. 327.

Guilloton, N. : Terminologie et communication, $n^{\circ} 2$, p. 215

Hendrickx, P. : Partial Dubbing, no 2, p. 217.

Houssat, J.-J. Lafitte : Utilisation du Science Citation Index en traduction, nº 3, p. 334.

Johnson, M. A. : Translation and Adaption, n' 4, p. 421.

Van Rutten, P. : La traduction de la Sophonisbe de Trissino, par Mellin de Saint-Gelais, $\mathrm{n}^{\circ} 2$, p. 224.

Voldeng, E. : La traduction poétique comme duplication ou dérivation textuelle d'une langue à une autre ?, $\mathrm{n}^{\circ} 2$, p. 220 


\section{Index français des mots et des sujets traités}

Abattoir traductionnel, n० 2, p. 143. Absorptivité, n², p. 198.

Accès au lexique, no 1, p. 69. Accessibilité des structures, no 1, p. 7. Accumulateur à pierres, $n^{\circ} 2$, p. 198. Acier intempérique, no 3 , p. 318 . Acier patinable, no 3 , p. 318 .

Acquisition du japonais, no 3, p. 285.

Actant dynamique, no 3, p. 260.

Actant statique, no 3, p. 260.

Acte de traduction, $n^{\circ} 1$, p. 31.

Actionnaire, no 3, p. 297.

Activité normalisatrice, $n^{\circ} 2$, p. 177.

Activité traduisante, n० 1, p. $44 ; n^{\circ} 2$, p. 144.

Adaptation culturelle, $n^{\circ} 3$, p. 266.

Affermage, $n^{\circ} 4$, p. 344.

AFNOR, no 2, p. 177.

Afrique noire, no 2, p. 139.

Agestion, $n^{\circ} 4$, p. 364 .

Agrammatisme, no $^{\circ}$, p. 106.

Agraphie pure, $n^{\circ} 1$, p. 13

Agrotechnie, no 3 , p. 282.

Aire de Broca, no 1 , p. $11 ; n^{\circ} 1$, p. 13.

Aire de Wernicke, no 1, p. 13.

Alcadiène, no 4 , p. 389.

Alcadiyne, n० 4 , p. 390.

Alcane, $n^{\circ} 4$, p. 388.

Alcatriène, no 4 , p. 389.

Alcène, no 4, p. 389 .

Alcényle, no 4, p. 389.

Alcyne, no 4, p. 390.

Alexie, $n^{\circ} 1$, p. 106.

Alexie-agraphie, no 1, p. 106.

Alkyle, no 4, p. 389.

Ambidextre, no 1, p. 18.

Amplificateur d'écoute, no 2, p. 194.

Analphabète, no 1, p. 22.

Analyse contextuelle, $n^{\circ} 3$, p. $286 ; n^{\circ} 3$, p. 287.

Analyse de l'Orset, no 2, p. 197.

Analyse du discours, no2, p. 144.

Analyse du discours comme méthode de traduction, $\mathrm{n}^{\circ} 2$, p. 144

Analyse ponctuelle, no 3, p. 286.

Anarthrie, n० 1, p. 106

Anarthrie pure, no 1 , p. $13 ; n^{\circ} 1$, p. 106.

Annotation de texte, $n^{\circ} 2$, p. 143

Anomalie comportementale, no 1, p. 10.

Anomie, no 1, p. 106.

Antiréflichissant, n०2, p. 199.
Aphasie, no 1, p. $10 ; n^{\circ} 1$, p. $11 ; n^{\circ} 1$, p. $41 ; n^{\circ} 1$, p. $68 ; n^{\circ} 1$, p. 106

Aphasie agrammatique, no 1, p. 106

Aphasie amnés(t)ique, $n^{\circ} 1$, p. 106.

Aphasie amnésique, no 1 , p. 16.

Aphasie anomique, no 1, p. 106.

Aphasie apraxique, no 1 , p. 106.

Aphasie d'expression, no 1, p. 106.

Aphasie d'impression, no 1, p. 106

Aphasie de Broca, no 1, p. $14 ; n^{\circ} 1$, p. 106.

Aphasie de compréhension, no 1, p. 106.

Aphasie de conduction, no 1, p. $15 ;$ no 1, p. 106.

Aphasie de réalisation phonématique, no 1 , p. 106.

Aphasie de "type Broca", no 1, p. 28.

Aphasie de Wernicke, no 1 , p. $14 ; n^{\circ} 1$, p. 106.

Aphasie de Wernicke avec prédominance de surdité verbale, $n^{\circ} 1$, p. 106

Aphasie de Wernicke du Type I, no 1, p. 106.

Aphasie de Wernicke du Type II, no 1, p. 106.

Aphasie de Wernicke du Type III, n* 1, p. 106.

Aphasie de Wernicke proprement dite, no 1, p. 106.

Aphasie du type A, no 1, p. 106.

Aphasie et traduction, no 1, p. 57.

Aphasie frontale, no 1, p. 106.

Aphasie globale, $n^{\circ} 1$, p. $17 ; n^{\circ} 1$, p. 106.

Aphasie mixte, $\mathrm{n}^{\circ} 1$, p. 17.

Aphasie motrice, no 1 , p. 106.

Aphasie motrice cinétique, $n^{\circ} 1$, p. 106.

Aphasie motrice corticale, $n^{\circ} 1$, p. 106.

Aphasie motrice efférente, no 1, p. 106.

Aphasie motrice périphérique, no 1, p. 106.

Aphasie motrice pure, no 1 , p. 106.

Aphasie motrice sous-corticale, no 1, p. 106.

Aphasie motrice transcorticale, $\mathrm{n}^{\circ} 1$, p. 106.

Aphasie non fluente, $\mathrm{n}^{\circ} 1$, p. 106.

Aphasie par lésion de la région du pli courbe, no 1 , p. 107.

Aphasie pragmatique, no 1, p. 107

Aphasie sensorielle, no 1, p. 107.

A phasie sensorielle corticale, $n^{\circ} 1$, p. 107.

Aphasie syntaxique, $n^{\circ} 1$, p. 107.

Aphasie temporale, $n^{\circ} 1$, p. 107.

Aphasie temporale à prédominance sensorielle, no 1, p. 107.

Aphasie transcorticale motrice, no 1 , p. 15.

A phasie transcorticale sensorielle, $\mathrm{n}^{\circ} 1$, p. 16 .

Aphasie verbale, no 1, p. 107.

Aphémie, no 1, p. 11 ; no 1, p. 107.

Appareil dialectique, $n^{\circ} 1$, p. 46 .

Appareil mains libres, no 2, p. 194.

Apport cognitif, $\mathrm{n}^{\circ} 3$, p. 287.

Apport linguistique, no 3 , p. 287.

Approximation, $n^{\circ} 3$, p. 247.

Apraxic, no 1, p. 107.

Apraxie articulatoire, no 1 , p. 107.

Apraxie verbale, no 1, p. 107.

Architecture solaire, $n^{\circ} 2$, p. 198.

Arène, no 4 , p. 391.

Arpon, $\mathrm{n}^{\circ} 2$, p. 184 .

Art didactique africain, $n^{\circ} 2$, p. 135 .

Articulation élastique, no 3, p. 282.

Artifice, $n^{\circ} 2$, p. 136.

Assemblage de cycle, no 4 , p. 392.

Assistant de langue, $n^{\circ} 2$, p. 176 .

Association, no 3 , p. 296 ; n० 3, p. 297. 
Associé, no 3, p. 297 ; no 3, p. 298.

Associé passif,, $n^{\circ} 3$, p. 298.

Assurances, $n^{\circ} 4$, p. 362.

Asymétrie fonctionnelle, $n^{\circ} 1$, p. 27.

Atome spirannique, $n^{\circ} 4$, p. 394

Attitude normative, $n^{\circ} 2$, p. 176

Audioconférence, $n^{\circ} 2$, p. 194.

Autocommutateur privé, n 2 , p. 194.

Autonomie, $n^{\circ} 4$, p. 350.

Autonomie neurofonctionnelle, $\mathrm{n}^{\circ} 1$, p. 66.

Bagage cognitif, no 3, p. 285 .

Bagage génétique, no 1, p. 29

Bagge, Christine, $n^{\circ} 2$, p. 135.

Ballon de stockage, no 2, p. 199.

Banque de terminologie, no 4 , p. 346 ; no 4 , p. 354

Base de l'opération de traduction, no 1, p. 5.

Bassnett-Mcguire, Susan, $n^{\circ} 2$, p. 135.

Beckett, no 3, p. 252.

Bégaiement, no 1, p. 41.

Bénard, J.-P., no 2, p. 144.

Bilingue, $\mathrm{n}^{\circ} 1$, p. 7.

Bilinguisme, no 1, p. 36.

Bioxyde d'azote, no 2, p. 197

Boîte aux lettres électronique, no 2, p. 194.

Boîte vocale, no 2, p. 194.

Broca, aire de, no 1, p. $11 ; n^{\circ} 1$, p. 13.

Broca, aphasie de, $\mathrm{n}^{\circ} 1$, p. 14.

Broca, aphasie de type, no 1, p. 28

Brossabilité, $\mathrm{n}^{\circ} 3$, p. 281.

Brown, modèle de Jason, $\mathbf{n}^{\circ} 1$, p. 29.

Brunet, Manon, $n^{\circ} 2$, p. 211.

Bureau des traductions, $n^{\circ} 2$, p. $202 ; n^{\circ} 4$, p. 341.

Bureautique, $n^{\circ} 4$, p. 348 .

But lucratif, $n^{\circ} 3$, p. 296.

Cabinet de traduction, no 4 , p. 353 .

Câble coaxial, no 2, p. 194.

Calage, $n^{\circ} 2$, p. 198.

Calcaire, no 3 , p. 316

Calque, $n^{\circ} 2$, p. $154 ; n^{\circ} 3$, p. 280.

Calque aménagé, no 3, p. 281.

Calque de forme, $n^{\circ} 3$, p. 281.

Calque littéral, no 3, p. 281.

Capteur, $\mathrm{n}^{\circ} 2$, p. $198 ; \mathrm{n}^{\circ} 2$, p. 199.

Capteur à air, no 2 , p. 198.

Capteur à concentration, no 2, p. 198.

Capteur plan, n $^{\circ} 2$, p. 199.

Capteur solaire, no 2, p. 199.

Caractère chinois, no 3 , p. 286

Carie verte, no 3 , p. 316.

Carroll, Lewis, no 3 , p. 266.

Cas clinique, $\mathrm{n}^{\circ} 1$, p. 38.

Cas oblique, $\mathrm{n}^{\circ} 2, \mathrm{p} .145$.

Catalogage, no 3 , p. 320.

Cécité verbale pure, no 1, p. 14.

Cendrars, $n^{\circ} 2$, p. 136.

Cerveau, no 1 , p. 6.

Cerveau bilingue, $\mathrm{n}^{\circ} 1$, p. 51

Cerveau du traducteur, $n^{\circ} 1$, p. $44 ; n^{\circ} 1$, p. 52.

Cerveau et langage, $\mathrm{n}^{\circ} 1$, p. 27.

Cerveau polyglotte, $n^{\circ} 1$, p. 51 .
Chariot à coke, no 3, p. 281.

Chat sauvage, $n^{\circ} 3$, p. 282 .

Chimie des composés du carbone, $n^{\circ} 4$, p. 387.

Chimie organique, no 4 , p. 387.

Chimiurgie, $n^{\circ} 3$, p. 282.

Clas, André, no 2, p. 211

Classe de pression, $\mathrm{n}^{\circ} 2$, p. 197.

Clavier à cames, $\mathbf{n}^{\circ} 2$, p. 197.

Clavier à fréquences vocales, no 2, p. 194.

Clavier à poussoir, no 2, p. 197.

Clavier décimal, no 2, p. 194.

Code, no 1 , p. 31 .

Coffret électronique, no 2, p. 194.

Cohérence, $\mathrm{n}^{\circ} 3$, p. 287.

Collecteur, $\mathbf{n}^{\circ} 2$, p. 198.

Collecteur à air, no 2, p. 198.

Collecteur plan, no 2, p. 199

Collecteur solaire, no 2, p. 199.

Colmatant, no 3 , p. 317.

Colpron, Gilles, no 2, p. 206.

Commande par programme enregistré, no 2, p. 194.

Commandité, no 3 , p. 298.

Commission ministérielle, $n^{0} 2$, p. 177.

Communication, norme de, no 2 , p. 175.

Commutation, $\mathbf{n}^{\circ} 2$, p. 123

Commutation de circuits, no 2, p. 194.

Commutation électronique, no 2, p. 194.

Commutation par paquets, $n^{\circ} 2$, p. 194.

Compagnie, no 3 , p. 296.

Compagnie privée, $n^{\circ} 3$, p. 299.

Compagnie publique, $n^{\circ} 3$, p. 299.

Compétence rédactionnelle, no 3, p. 285.

Comportement verbal, no 1 , p. 6.

Compos, $\mathrm{n}^{\circ} 2$, p. 194.

Composante corticale, no 1, p. 11.

Compréhensibilité du texte, $n^{\circ} 2$, p. 179.

Compte-mots, $n^{\circ} 4$, p. 349.

Concentrateur, $n^{\circ} 2$, p. 198.

Conception wernickéenne, $n^{0} 1$, p. 10 .

Conceptualisation, $\mathrm{n}^{\circ} 1$, p. 71 .

Conférence à trois, no 2 , p. 194.

Conférence textuelle assistée par ordinateur, $\mathrm{n}^{\circ} 2$, p. 194.

Connaissance spécialisée, no 3 , p. 285.

Connexion, $n^{\circ} 1$, p. $64 ; n^{\circ} 1$, p. 66.

Conte africain, $\mathrm{n}^{\circ} 2$, p. 135.

Conte folklorique, $\mathrm{n}^{\circ} 2$, p. 135 .

Conte traditionnel africain, $\mathbf{n}^{\circ} 2$, p. 135 .

Contenu, plan du, $\mathrm{n}^{\circ} 2$, p. 123 .

Contes d'Amadou Koumba, $n^{\circ} 2$, p. 135.

Conteur traditionnel, no 2, p. 137.

Conversion photothermique, $\mathbf{n}^{\circ} 2$, p. 199

Coréférentialité, $n^{\circ} 3$, p. 261.

Corporation, no 3, p. $296 ; n^{\circ} 3$, p. 300.

Correspondance, $\mathrm{n}^{\circ} 3$, p. 247.

Cortex interprétatif, $\mathrm{n}^{\circ} 1$, p. 53.

Cosmologique, $n^{0} 3$, p. 262.

Costa, modèle de, no 1, p. 30.

Couche antiréflecteur, $\mathrm{n}^{\circ} 2$, p. 199.

Couche d'atelier, no 3, p. 317

Couche sélective, no 2, p. 199

Courant vagabond, $n^{\circ} 3$, p. 317.

Courrier électronique, $\mathrm{n}^{\circ} 2$, p. 194.

Cours de traduction, $n^{\circ} 2$, p. 143.

Coût fixe marginal, n० 3 , p. 308. 
Coût marginal, no 3, p. 306.

Création linguistique, $\mathrm{n}^{\circ} 3$, p. 280. Cuve d'accumulation, no 2, p. 199.

Cyclane, no 4 , p. 391.

Cycle condensê, no 4 , p. 392

Cyclène, $n^{\circ} 4$, p. 391 .

Cyclo-oléfine, no 4 , p. 391.

Cycloaclcyne, no 4, p. 391.

Cycloalcane, $n^{\circ} 4$, p. 391 .

Cycloalcène, no 4, p. 391.

Cycloparaffine, no 4, p. 391.

Débit de combustible, no 2, p. 198.

Découverte anatomoclinique, $\mathrm{n}^{\circ} 1$, p. 11

Déficit de lexicalisation, $n^{0} 1$, p. 74.

Degré de systématisation, $n^{\circ} 1$, p. 31 .

Degré de technicité, no ${ }^{\circ}$, p. 178.

Delisle, Jean, $\mathrm{n}^{\circ} 2$, p. 144.

Démarche onomasiologique, $\mathrm{n}^{\circ} 1$, p. 45.

Démarche sémasiologique, $\mathrm{n}^{\circ} 1$, p. 45 .

Dérivation textuelle, $n^{\circ} 2$, p. 220 .

Désintégration phonétique, $n^{\circ} 1$, p. 107.

Désir de traduire, $\mathrm{n}^{\circ} 3$, p. 246.

Diagramme température-concentration, no 3 , p. 317. Facilitation sémantique, $n^{\circ} 1$, p. 70

Dialectisation, no 3, p. 261.

Dialogue, $n^{\circ} 2$, p. 139.

Dictionnaire de l'eau, no 4, p. 407

Dictionnaire des anglicismes, $n^{\circ} 2$, p. 206

Diène, no 4 , p. 389.

Différence interindividuelle, $\mathrm{n}^{\circ} 1$, p. 32.

Digression, $n^{\circ} 2$, p. 137.

Digression-commentaire, n० 2, p. 137.

Dimension chronobiologique, no 1 , p. 28.

Dioléfine, no 4 , p. 389.

Diop, Birago, $\mathrm{n}^{\circ} 2$, p. 135.

Discours, analyse du, $n^{\circ} 2$, p. 144

Discours de spécialité, no 2 , p. 175

Disponibilité immédiate, no 1, p. 7.

Dissyntaxie, no 1 , p. 107.

Documentation, $n^{\circ} 4$, p. 345 .

Domaine d'application, no 2, p. 177.

Domestication de l'informatique, $n^{0} 4$, p. 367.

Duplication, no 2, p. 220.

Dysarthrie corticale, $\mathrm{n}^{\circ} 1$, p. 107.

Dysfonction, no 1, p. 13 .

Dysgraphie d'évolution, $n^{\circ} 1$, p. 107.

Dyslexie d'évolution, $\mathrm{n}^{\circ} 1$, p. 107.

Dysphasie d'évolution, no 1 , p. 107.

Dyspraxie, no 1, p. 107.

Dysprosodie, no 1 , p. 107.

Eau piégée, no 3, p. 281

Ecoute dichotique, $n^{\circ} 1$, p. 31 .

Écrire et traduire, no 4, p. 409

Écriture, $n^{\circ} 3$, p. 256.

Effet de serre, no 2, p. 199.

Égohine, $\mathrm{n}^{\circ} 2$, p. 184 .

Égoïne, $n^{\circ} 2$, p. $184 ; n^{\circ} 2$, p. 185.

Egoïne à refendre, $\mathrm{n}^{\circ} 2$, p. $182 ; \mathrm{n}^{\circ} 2$, p. $185 ; \mathrm{n}^{\circ} 2$, p. 186.

Égoïne à tronçonner, $n^{\circ} 2$, p. $182 ; n^{\circ} 2$, p. 185

Égoïne de débit, no 2 , p. 185.

Genérateur mural, n² 2, p. 198.
Électron de valence, $n^{\circ} 4$, p. 387.

Éliminateur d'oxygène, no 3, p. 317

Émail de brai de houille, no 3, p. 316.

Émissivité, no 2, p. 199.

Émittance, $n^{\circ} 2$, p. 199.

Emploi des calques, no 3, p. 282.

Emprunt hybride, $n^{\circ} 3$, p. 280.

Emprunt intégral, nº 3 , p. 280.

Emprunt linguistique, $\mathrm{n}^{\circ} 3$, p. 280.

Emprunt partiel, no 3, p. 280.

Emprunt remanié, no 3, p. 280.

Encodage du message, $\mathrm{n}^{\circ} 1$, p. 66

Enseignement de la traduction, no 2, p. 143.

Enseignement standard, $n^{\circ} 1, p .10$.

Entrée en interaction, no 1 , p. 6

Entreprise marginale, no 3, p. 306.

Esprit animiste, no 2, p. 139.

Éthique de la profession, no 4, p. 352.

Exégèse textuelle, $n^{\circ} 2, p .146$.

Exemple de Plaute, n० 3 , p. 246.

Explication de texte, $\mathrm{n}^{\circ} 2$, p. 144

Expression équivalente, $\mathrm{n}^{\circ} 1$, p. 66.

Facteur com

Facteur d'air, no 2, p. 196

Facteur fumivore, $n^{\circ} 2$, p. 197

Faisceau associatif, $\mathrm{n}^{\circ} 1$, p. 12

Fautes, typologie des, no 2, p. 146

Feuillet à poing, $n^{\circ} 2$, p. 184.

Fiabilité linguistique, no 3 , p. 288.

Fibre associative, no 1 , p. 12

Fibre optique, ${ }^{\circ} 2$, p. 194

Focalisateur, no 2, p. 198.

Fonction cognitive, no 1, p. 31.

Fonction linguistique, no 1 , p. 30 .

Fonction musicale, $n^{\circ} 1$, p. 31 .

Fonction publique, $n^{\circ} 4$, p. 341 .

Fonction régulatrice du langage, $n^{\circ} 1$, p. 55

Fonctionnement cérébral, modèle de, $\mathrm{n}^{\circ} 1$, p. 32

Fonctionnement modulaire, $n^{\circ} 1$, p. 10.

Formation, $\mathrm{n}^{\circ} 4$, p. 347.

Forme verbale, $\mathrm{n}^{\circ} 2, \mathrm{p} .179$.

Fournisseur d'information, $n^{\circ} 2$, p. 194

Franglais, no 4 , p. 416.

Gairaigo, n० 3, p. 287.

Gaucher, $n^{\circ}$ 1, p. 18.

Gérant, no 3, p. 298.

Gestion et traduction, no 4 , p. 340 .

Gestionnaire, $n^{\circ} 4$, p. 341

Godendard, $n^{\circ} 2$, p. 183.

Godendard pour un seul homme, n ${ }^{\circ} 2$, p. 184.

Godendart, no 2, p. 184.

Goldberg, modèle de, $\mathrm{n}^{\circ} 1$, p. 30.

Grammaire générative, $n^{\circ} 1$, p. 46.

Grammaire universelle, $n^{\circ}$ 1, p. 46.

Grande aphasie de Broca, $\mathrm{n}^{\circ}$ 1, p. 107.

Grande aphasie de Wernicke, no 1, p. 107.

Griot, $n^{\circ} 2$, p. 135.

Guide de la traduction appliquée, no 2, p. 144 
Guide du rédacteur de l'administration fédérale, $n^{\circ} 2$, p. 202.

Gyrus angulaire, $n^{\circ} 1$, p. 11.

Gyrus supramarginal, $n^{\circ} 1$, p. 11.

Héliotechnique, no 2, p. 199.

Hémisphère cérébral, $\mathrm{n}^{\circ} 1, \mathrm{p} .11$.

Hémisphère gauche, $\mathrm{n}^{\circ} 1$, p. 27.

Heschl, zone de, $n^{\circ} 1$, p. 13.

Homologue, no 4, p. 388.

Horguelin, $P, n^{\circ} 2$, p. 144.

Hutchinson, J.A., n ${ }^{\circ} 2$, p. 135

Hydrocarbure, $n^{\circ} 4$, p. 387.

Hydrocarbure acétylénique, no 4, p. 390.

Hydrocarbure acyclique, no 4 , p. 388.

Hydrocarbure alicyclique, no 4, p. 391.

Hydrocarbure aliphatique, $n^{0} 4$, p. 388.

Hydrocarbure aromatique, n० 4, p. 391.

Hydrocarbure cyclique, $n^{\circ} 4$, p. 391.

Hydrocarbure éthylénique, $\mathrm{n}^{\circ} 4$, p. 389.

Hydrocarbure oléfine, $n^{\circ} 4$, p. 389.

Hydrocarbure polycyclique condensé, no 4, p. 392.

Hydrocarbure ponté, no 4, p. 393.

Hydrocarbure spirannique, $n^{\circ} 4$, p. 394.

Identification, $n^{\circ} 2$, p. 177.

Imagerie traditionnelle, $n^{\circ} 2$, p. 139.

Importance des notes, $\mathrm{n}^{\circ} 2, \mathrm{p} .144$.

Impossibilité de l'équivalence, $n^{\circ} 3$, p. 247.

Inclinaison du collecteur, $\mathrm{n}^{\circ} 2$, p. 198.

Incorporée, no 3 , p. 300 .

Indépendance neurobiologique, $\mathrm{n}^{\circ} 1, \mathrm{p} .6$.

Information linguistique, $n^{\circ} 1$, p. 5 .

Informatique, $\mathrm{n}^{\circ} 2$, p. 208.

Informatique, domestication de, no 4, p. 367.

Ingénierie solaire, no 2, p. 199.

Inhibition, no 1, p. 7.

Insolateur, no 2, p. 198.

Interrogations, type $d^{\prime}, n^{\circ} 4$, p. 371.

Intertextualité, n 3, p. 252.

Japonais-français, $\mathrm{n}^{\circ} 3$, p. 285.

Jiongo, no 3 , p. 287.

Jonction spirannique, $n^{\circ} 4$, p. 394.

Katakana, no 3, p. 287.

Kocourek, Rostislav, no 2, p. 209.

Langage performé, $n^{\circ} 2$, p. 175.

Langue $1, n^{\circ} 1$, p. 5 .

Langue $2, n^{0} 1$, p. 5.

Langue agglutinante, $n^{\circ} 1$, p. 19.

Langue cible, $n^{\circ} 2$, p. 180.

Langue de spécialité, n० 2, p. 208.

Langue écrite, no 1, p. 21.
Langue française de la science et de la technique, $n^{\circ} 2$, p. 209.

Langue polysynthétique, $\mathrm{n}^{\circ} 1$, p. 19.

Langue seconde, $\mathrm{n}^{\circ} 1$, p. 31 .

Langue tonale, $\mathrm{n}^{\circ} 1$, p. 20.

Langues africaines, $n^{\circ} 2$, p. 212.

Larose, Robert, $\mathrm{n}^{\circ} 2$, p. 143.

Latéralisation fonctionnelle, $\mathrm{n}^{\circ} 1$, p. 20

Latéralisé, $\mathrm{n}^{\circ} 1$, p. 27 ; $\mathrm{n}^{\circ} 1$, p. 30 .

Lésion sous-corticale, $\mathrm{n}^{\circ} 1$, p. 13.

Lésions, no 1 , p. 10

Lessive de potasse, no 2 , p. 197.

Lethuillier, Jacques, no 2, p. 208.

Lexicalisation, déficit de, $n^{\circ} 1$, p. 74

Lexicalisation et traduction, $\mathrm{n}^{\circ} 1$, p. 78 .

Lexicalisation, processus de, $\mathrm{n}^{\mathrm{o}} 1$, p. 68

Lexie, $\mathrm{n}^{\circ} 2$, p. 180

Lexie fixée, $n^{\circ} 2$, p. 180 .

Lexique, accès au, no 1, p. 69

Lexique mental, $\mathrm{n}^{\circ} 1$, p. 69.

Lexique mental, organisation du, no 1, p. 69 .

Liaison phonosémantique, $n^{\circ} 3$, p. 264.

Limitée, $\mathrm{n}^{\circ} 3$, p. 300

Littérature orale, $\mathrm{n}^{\circ} 2$, p. 140.

Lobule pariétal, no 1 , p. 12

Localisation anatomique, $\mathrm{n}^{\circ} 1$, p. 10

Logiciel de messagerie, $n^{\circ} 2$, p. 194.

Loi sur les langues officielles, $n^{\circ} 4$, p. 341.

Machine de traitement de textes, $n^{\circ} 4$, p. 348.

Manque du mot, $\mathbf{n}^{\circ} 1$, p. 107.

Marginal, no 3, p. 306.

Marginaliste, $n^{\circ} 3$, p. 306

Matrice multidimensionnelle, $\mathrm{n}^{\circ} 1$, p. 54.

Mécanique des sols, $n^{\circ} 4$, p. 410

Mécanisme du calque, $n^{\circ} 3$, p. 281.

Mélange eutectique, $n^{\circ} 2$, p. 199.

Message, encodage du, no 1 , p. 66

Messagerie textuelle, $n^{\circ} 2$, p. 194.

Messagerie vocale, $n^{\circ} 2$, p. 194

Micro-ordinateur, $n^{\circ} 4$, p. 367.

Micros-banque de terminologie, $n^{\circ} 4$, p. 367 .

Militante féminine, $n^{\circ} 2$, p. 219.

Modèle, $n^{\circ} 2$, p. 176 .

Modèle de comportement, $\mathbf{n}^{\circ} 2$, p. 175.

Modèle de Costa, $n^{\circ} 1$, p. 30.

Modèle de fonctionnement cérébral, $n^{\circ} 1$, p. 32.

Modèle de Goldberg, no 1, p. 30.

Modèle de Jason Brown, no 1, p. 29

Modèle isotopique, $n^{\circ} 3$, p. 254 .

Modèle linguistique, $n^{\circ} 1$, p. $45 ; n^{\circ} 2$, p. 175

Modèle psycholinguistique, $n^{\circ} 1$, p. 68 .

Modulation, $n^{\circ} 2$, p. 151 .

Mouvement, $n^{\circ} 3$, p. 256.

Multidiffusion, $\mathrm{n}^{\circ}$ 2, p. 194

Mutisme verbal, $n^{\circ} 1$, p. 107

Mutisme verbal pur, $\mathrm{n}^{\circ} 1$, p. 107.

Natif, $n^{\circ} 2$, p. 176

Néologisme, no 1, p. 77.

Neurolinguistique, psycholinguistique et traduction, no 1, p. 5. 
Neurolinguistique, terminologie de la, $n^{0} 1$, p. 91 .

Neurophysiologie, $\mathbf{n}^{\circ} 1$, p. 36.

Neurophysiologue, no 1 , p. 49

Niveau conceptuel, no 1, p. 71

Niveau de profondeur, $n^{\circ} 1$, p. 7.

Nom propre, $n^{\circ} 3$, p. 267

Nominalisation, $\mathrm{n}^{\circ} 2$, p. 179

Noms propres, traduction des, $n^{\circ} 2$, p. 180

Noologique, $\mathrm{n}^{\circ} 3$, p. 262.

Norme, $\mathrm{n}^{\circ} 2, \mathrm{p}, 175$

Norme de communication, $n^{\circ} 2$, p. 175

Note encyclopédique, $\mathrm{n}^{\circ} 2$, p. 146.

Note linguistique, $\mathrm{n}^{\circ} 2$, p. 146

Note traductionnelle, $n^{\circ} 2$, p. 146.

Notes, importance des, no 2, p. 144

Notes, typologie des, $n^{\circ} 2$, p. 145.

Noyau benzénique, $n^{\circ} 4$, p. 391.

Numérotation abrégée, no 2 , p. 194.

Opération traduisante, $\mathrm{n}^{\circ} 1$, p. 48

Organisation du lexique mental, no 1, p. 69.

Paradigme des actants, $n^{\circ} 3$, p. 260.

Paraffine, $n^{\circ} 4$, p. 388

Paragrammatisme, no 1, p. 107.

Paragraphie, $n^{\circ} 1$, p. 107.

Paralexie, no 1, p. 107.

Paraphasie, $\mathrm{n}^{\circ} 1$, p. 107

Paraphasie-phonémique, $\mathrm{n}^{\circ} 1$, p. 77.

Paraphasie sémantique, $n^{\circ} 1$, p. 77

Paraphasie verbale formelle, $\mathrm{n}^{\circ} 1$, p. 77.

Part sociale, $n^{\circ} 3$, p. 299.

Passe par tout, $\mathrm{n}^{\circ} 2$, p. 184

Passe-partout, $n^{\circ} 2$, p. $182 ; n^{\circ} 2$, p. $183 ; n^{\circ} 2$, p. 187.

Passepartout, $\mathrm{n}^{\circ} 2$, p. 184

Passerelle, no 2, p. 194.

Passif social, no 3 , p. 298.

Patient polyglotte, no 1 , p. 6 .

Patte de chien, no 3 , p. 281

Peinture d'attente, no 3 , p. 317.

Perchiste, $\pi^{\circ} 2$, p. 177

Périphrase, no 1, p. 77.

Péritéléphonie, $\mathrm{n}^{\circ} 2$, p. 194

Permutation, $n^{\circ} 3$, p. 248.

Peroxyde d'azote, $\mathrm{n}^{\circ} 2$, p. 197.

Personne morale, $n^{\circ} 3$, p. $296 ; n^{\circ} 3$, p. 297.

Perturbation, $n^{\circ} 1$, p. $6 ; n^{\circ} 1$, p. 13.

Phallocrate, $n^{\circ} 2$, p. 219.

Phrase-transition, $n^{\circ} 2$, p. 137.

Phrénologie des bosses, $\mathrm{n}^{\circ} 1$, p. 10.

Phrénologie des circonvolutions, $n^{\circ} 1$, p. 10.

Plan du contenu, no 2, p. 123.

Plan final, $n^{\circ} 3$, p. 282.

Poésie, no 3, p. 258.

Politique d'affermage, no 4, p. 344

Polyène, $n^{\circ} 4$, p. 389.

Polyglotte, $n^{\circ} 1$, p. $6 ; n^{\circ} 1$, p. $20 ; n^{\circ} 1$, p. 38.

Posemètre automatique, $n^{\circ} 3$, p. 282

Poste (téléphonique) à cadran, no 2, p. 194.

Poste (téléphonique) à clavier, $n^{\circ} 2$, p. 195.

Poste à clavier multifréquence, $n^{\circ} 2$, p. 195.
Pouvoir comburivore, $n^{\circ} 2$, p. 196.

Pratique de la traduction, $\mathrm{n}^{\circ} 2$, p. 144.

Première circonvolution, $\mathrm{n}^{\circ} 1, \mathrm{p} .11$.

Primaire après grenaillage, no 3 , p. 317.

Primaire d'atelier, $n^{\circ} 3$, p. 317.

Problème culturel, no 3 , p. $266 ; n^{\circ} 3$, p. 279.

Processeur graphique, no 2, p. 195.

Processus de lexicalisation, $n^{\circ} 1$, p. 68 .

Processus neuropsycholinguistique, $n^{\circ} 1$, p. 5.

Professionnalisme, $n^{\circ} 4$, p. 350 .

Profil superficiel, $\mathrm{n}^{\circ} 3$, p. 316.

Pseudolus, no 3, p. 246.

Psychomécanique, no 1, p. 48.

Psychosystématique, no 1 , p. 48

Pupitre dirigeur, n² 2, p. 195.

Qualité créatrice, no 2, p. 135.

Radical bivalent, $n^{\circ} 4$, p. 390

Radical plurivalent, no 4 , p. 390

Radical trivalent, $n^{\circ} 4$, p. 390.

Radioalignement de piste, no 3, p. 282.

Raison sociale, $\mathrm{n}^{\circ} 3$, p. 300 .

Rappel automatique, $n^{\circ} 2$, p. 195.

Raquette, $n^{\circ} 2$, p. $182 ; n^{\circ} 2$, p. 183.

Recherche d'un équivalent, $\mathrm{n}^{\circ} 3$, p. 247.

Recherche indirecte, $n^{\circ} 3$, p. 289.

Recherche terminologique, $n^{\circ} 3$, p. 285.

Recherche terminologique, trois niveaux de la, $n^{\circ} 3$, p. 286.

Récréation poétique, $\mathrm{n}^{\circ} 2$, p. 221.

Recrutement, no 4, p. 347 .

Réduction horizontale, $\mathrm{n}^{\circ} 3$, p. 260

Réduction verticale, $\mathrm{n}^{\circ} 3$, p. 260.

Registre délocutif, $\mathrm{n}^{\circ} 3$, p. 261.

Relation métaphorique, $n^{\circ} 3$, p. 283.

Relief d'ancrage, no 3, p. 316.

Remplissage pittoresque, $n^{\circ} 2$, p. 137.

Renaissance italienne, $n^{\circ} 2$, p. 224.

Rentabilité, $n^{\circ} 4$, p. 342.

Renvoi automatique (d'appels), no 2, p. 195.

Répondeur à interrogation à distance, $\mathrm{n}^{\circ} 2$, p. 195.

Répondeur-enregistreur, $\mathrm{n}^{\circ} 2$, p. 195.

Répondeur interrogeable, $n^{\circ} 2$, p. 195.

Répondeur simple, $n^{\circ} 2$, p. 195.

Répondeur téléphonique, no 2, p. 195.

Réseau de règles, $n^{\circ} 2$, p. 175.

Réseau en anneau, no 2 , p. 195.

Réseau en bouche, $n^{\circ} 2$, p. 195.

Réseau en bus, $n^{\circ} 2$, p. 195.

Réseau en étoile, $n^{\circ} 2$, p. 195.

Réseau local (d'entreprise) (RLE), nº 2, p. 195.

Réseau neuronique, $\mathrm{n}^{\circ} 1$, p. 30 .

Réseau numérique à intégration des services (RNIS), no 2, p. 195.

Réseau télégraphique, $\mathrm{n}^{\circ} 2$, p. 195.

Réseau téléphonique, $\mathrm{n}^{\circ} 2$, p. 195.

Réservoir, $n^{\circ} 2$, p. 199.

Responsabilité illimitée, no 3, p. 296

Responsabilité limitée, $n^{\circ} 3$, p. 297.

Ressource technique, $n^{\circ} 4$, p. 354.

Ressources humaines, no 4, p. 352 
Revêtement antireflet, $\mathrm{n}^{\circ}$ 2, p. 199

Revêtement sélectif, $\mathbf{n}^{\circ} 2$, p. 199.

Rezeptionsforschung, no 3, p. 252.

Richesses et particularités de la langue écrite au Québec, no 2, p. 211.

Routinisation, $\mathrm{n}^{\circ} 1$, p. 29

Rugosité d'accrochage, $n^{\circ} 3$, p. 316.

Rugosité superficielle, $n^{\circ} 3$, p. 316.

Sarzan, no 2, p. 135

Saturé, no 4 , p. 387.

Savoir lexical, no 1, p. 75.

Scie à chevilles, no 3, p. 293.

Scie à dos, no 3, p. 291.

Scie à dos renforcé, $n^{\circ} 3$, p. 290.

Scie à dosseret, $\mathrm{n}^{\circ} 3$, p. 291

Scie à dossière, $\mathrm{n}^{\circ} 3$, p. 291.

Scie à élagueur, no 2, p. 188.

Scie à émonder, $n^{\circ} 2$, p. 188.

Scie à greffer, $\mathrm{n}^{\circ} 2$, p. $182 ; \mathrm{n}^{\circ} 2$, p. $185 ; \mathrm{n}^{\circ} 2$, p. 186.

Scie à guichet, $n^{\circ} 2$, p. $182 ; n^{\circ} 2$, p. $185 ; n^{\circ} 2$, p. 187.

Scie à main, $n^{\circ} 2$, p. $182 ; n^{\circ} 2$, p. 184

Scie à onglet, $\mathrm{n}^{\circ} 3$, p. 292.

Scie à panneau, no 2 , p. $182 ; n^{\circ} 2$, p. $185 ; n^{\circ} 2$, p. 187.

Scie à panneaux, no 2, p. 187.

Scie à parquet, $n^{\circ} 3$, p. 294.

Scie à placage, $n^{\circ} 3$, p. 294

Scie à poignée, $\mathrm{n}^{\circ} 2, \mathrm{p} .182$

Scie à poignet, $\mathrm{n}^{\circ} 2$, p. 182 .

Scie à queue d'aronde, $\mathrm{n}^{\circ} 3$, p. 291

Scie à recaler, $\mathbf{n}^{\circ} 3$, p. 294.

Scie à refendre, $\mathrm{n}^{\circ} 2$, p. 186

Scie à renfort, no $^{\circ}$, p. 290.

Scie à renfort métallique, no 3, p. 291.

Scie à tronçonner, $n^{\circ} 2$, p. 185 .

Scie à voleur, $n^{\circ} 2$, p. 187.

Scie baïonnette, $\mathrm{n}^{\circ} 2$, p. 187

Scie d'ébéniste, no 3, p. 292

Scie d'élagage, $\mathrm{n}^{\circ} 2$, p. 188.

Scie d'encadrement, $n^{\circ} 3$, p. 291.

Scie d'encadreur, $n^{\circ} 3$, p. 291.

Scie de jardinier, $\mathrm{n}^{\circ} 2$, p. $182 ; \mathrm{n}^{\circ} 2$, p. $185 ; \mathrm{n}^{\circ} 2$, p. 188 .

Scie de long, $n^{\circ} 2$, p. $182 ; n^{\circ} 2$, p. 183 .

Scie de travers, $\mathrm{n}^{\circ} 2$, p. 185

Scie défonceuse, no 2 , p. $182 ; n^{\circ} 2$, p. 188.

Scie égohine, $n^{\circ} 2$, p. 184

Scie-egoīne, $n^{\circ} 2$, p. 184.

Scie harpon, no 2 , p. 184.

Scie hégoïne, no 2, p. 184

Scie manuelle, no 4 , p. 395.

Scie passe-partout, $n^{\circ} 2$, p. $182 ; n^{\circ} 2$, p. $183 ; n^{\circ} 2$, p. 185

Scie passe-partout à deux mains, $n^{\circ} 2$, p. 184.

Scie passe-partout type égoïne, no 2, p. 184.

Scie pour boîte à coupe, no 3 , p. 294.

Scie raquette, no 2, p. 183.

Scie renforcée, no 3, p. 290.

Scie renvoyée, no 3, p. 293.

Scie sans monture, $\mathrm{n}^{\circ} 2$, p. 182.
Scie Sterling, no 3, p. 292

Scripte, $n^{\circ} 2$, p. 177.

Secrétariat d'État, $n^{\circ} 2$, p. 202.

Secteur privé, $n^{\circ} 4, p .341$

Secteur public, no 3 , p. $300 ; n^{\circ} 4$, p. 341

Segmentation, $n^{\circ} 2$, p. 146.

Sémantique structurale, $n^{\circ} 2$, p. 123.

Sémiotique structurale, $n^{\circ} 3$, p. 252.

Senghor, $n^{\circ} 2$, p. 139

Sérialisation spatiale, no 3 , p. 262

Série homologue, $n^{\circ} 4$, p. 388 .

Seuil de contrainte de non-rupture, no 3 , p. 318 .

Seutin, Émile, $n^{\circ} 2$, p. 211.

Sexiste, $n^{\circ} 2$, p. 219

Sigle, $n^{\circ} 2$, p. 180

Signe canonique, $\mathrm{n}^{\circ} 1$, p. 5 .

Siliciuration, $\mathrm{n}^{\mathrm{O}} 3$, p. 317

Site, no 1 , p. 40

Société, no 3, p. 296.

Société anonyme, no 3, p. 299.

Société civile, $\mathrm{n}^{\circ} 3$, p. 297.

Société commerciale, $\mathrm{n}^{\circ} 3$, p. 297

Société de capitaux, no 3, p. $297 ; n^{\circ} 3$, p. 298

Société de frais, no 3, p. 301.

Société de moyens, no 3 , p. 301

Société de personnes, no 3, p. 297.

Société en commandite, no 3, p. $297 ; n^{\circ} 3$, p. 298.

Société en nom collectif, no 3, p. 297

Sociolecte, $n^{\circ} 3$, p. 285.

Solarchitecture, $\mathrm{n}^{\circ} 2$, p. 198

Solution eutectique, $\mathrm{n}^{\circ} 2$, p. 199.

Solution, stratégie de, $n^{\circ} 1$, p. 33

Sondage d'exploration, $n^{\circ} 3$, p. 282

Sophonisbe, $\mathrm{n}^{\circ} 2$, p. 224.

Source indirecte, $\mathrm{n}^{\circ} 3$, p. 289.

Spinelle, no 3, p. 317.

Stockage, $n^{\circ} 2$, p. 198.

Stockage à lit de roches, $\mathrm{n}^{\mathrm{0}} 2$, p. 199

Stockage de courte durée, no 2, p. 199.

Stockage de longue durée, $n^{\circ} 2$, p. 199

Stockage intersaisonnier, $\mathrm{n}^{\circ} 2$, p. 199.

Stratégie de solution, no 1 , p. 33.

Stratégie de traduction, no 1, p. 66.

Structure biologique, $n^{0} 1$, p. 6 .

Structure cérébrale, $n^{\circ} 1$, p. 40.

Studio de conférence, $n^{\circ} 2$, p. 195.

Substance psychique, $n^{\circ} 1$, p. 5.

Substantiver, $n^{\circ} 2$, p. 181 .

Substrat biologique, $n^{\circ} 1$, p. 6 .

Support élastique, $\mathrm{n}^{\circ} 3$, p. 282.

Support somatique du langage, $n^{\circ} 1$, p. 49

Surdité verbale pure, $n^{\circ} 1$, p. 13.

Syndrome d'isolement (de l'aire du langage), $n^{\circ} 1$, p. 107.

Syndrome de désintégration phonétique, $n^{\circ} 1$, p. 107.

Syndrome pariéto-pli courbe, no 1, p. 107.

Systématibilité, $\mathrm{n}^{\circ}$ l, p. 29.

Systématisation, degré de, $\mathrm{n}^{\circ} 1$, p. 31.

Système actantiel, no 3 , p. 262.

Système de messagerie textuelle, $n^{\circ} 2$, p. 195.

Système de messagerie vocale, $n^{\circ} 2$, p. 195.

Système de modélisation, $n^{\circ} 3$, p. 258.

Système de téléécriture, $n^{\circ} 2$, p. 195.

Système nerveux, $\mathrm{n}^{\circ} 1$, p. 36 . 
Système neurofonctionnel, no 1 , p. $64 ; n^{0} 1$, p. 66. Système "TERM.X", no 4, p. 367.

Système terminologique, $n^{\circ} 4$, p. 367.

Système vidéotex, $\mathrm{n}^{\circ} 2, \mathrm{p} .195$.

Systran, $n^{\circ} 2$, p. 178.

Tablette de téléécriture, $n^{\circ} 2$, p. 195.

Tartre calcaire, no 3 , p. 316 .

Taux d'aération, no 2, p. 196

Technicité, degré de, no 2 , p. 178.

Technique numérique, $\mathrm{n}^{\circ} 2$, p. 195.

Téléconférence, $n^{\circ} 2$, p. 195.

Téléconférence audiographique, no 2, p. 195.

Téléconférence informatisée, $n^{\circ} 2$, p. 195.

Télécopie, nº 2, p. 195.

Télécopieur, no 2, p. 195.

Téléimprimeur, $\mathrm{n}^{\mathrm{0}} 2$, p. 195.

Télématique, $n^{\circ} 4$, p. 355 .

Téléterminal, no 2, p. 195.

Télétex, $n^{\circ} 2$, p. 195.

Télétexte, no 2, p. 195.

Télex, no 2, p. 195.

Temps réel, $\mathrm{n}^{\circ} 1$, p. 8

Terme nouveau, no 3 , p. 319

Terminal intelligent, $n^{\circ} 2$, p. 195

Terminologie, $n^{\circ} 2$, p. $177 ; n^{\circ} 4$, p. 345 .

Terminologie aphasiologique, $n^{\circ} 1$, p. 97.

Terminologie automatisée, $n^{\circ} 4$, p. 372 .

Terminologie de la neurolinguistique, $\mathrm{n}^{\circ} 1$, p. 91 .

Terminologie et communication, $n^{\circ} 2$, p. 216 .

Terminologie, micro-banques de, no 4 , p. 367 .

TERMIUM I, n' 4 , p. 346.

TERMIUM II, no 4, p. 346.

TERMIUM III, no 4, p. 346 .

Texte, explication de, $\mathrm{n}^{\circ} 2$, p. 144

Texte japonais, $n^{\circ} 3$, p. 285 .

Texte poétique, no 3 , p. 258 .

Texte pragmatique, $n^{\circ} 2$, p. 143.

Texte technique, $\mathrm{n}^{\circ} 2, \mathrm{p} .175$.

Textverarbeitung, $n^{\circ} 3$, p. 252

Théorie guillaumienne du langage, $n^{0} 1$, p. 48.

Thermomètre à dilatation, $n^{\circ} 2$, p. 197.

Touche, $n^{\circ} 2$, p. 195.

Tradition, $\mathbf{n}^{\circ} 2$, p. 139.

Traduction à vue, no 2, p. 143.

Traduction, acte de, $n^{\circ} 1$, p. 31 .

Traduction assignée par ordinateur, $n^{\circ} 4$, p. 355 .

Traduction automatique, $n^{\circ} 2$, p. $178 ; n^{\circ} 4$, p. 349 .

Traduction, base de l'opération de, $\mathrm{n}^{\circ} 1, \mathrm{p} .5$.

Traduction brute, $n^{\circ} 2$, p. 178.

Traduction courante, $\mathrm{n}^{\circ} 2$, p. 220

Traduction, cours de, $n^{\circ} 2$, p. 143.

Traduction des noms propres, $\mathrm{n}^{\circ} 2$, p. 180.

Traduction, enseignement de la, $n^{\circ} 2, p .143$.

Traduction et création, $\mathrm{n}^{\circ} 2$, p. 135 .

Traduction exacte, $n^{\circ} 2$, p. 220.

Traduction japonais-français, $n^{\circ} 3$, p. 285.

Traduction littéraire, n० 3 , p. $246 ; n^{\circ} 3$, p. 252.

Traduction poétique, $n^{\circ} 2$, p. 220 .

Traduction scientifique et technique, $n^{\circ} 3$, p. 285.

Traduction, stratégie de, $n^{\circ} 1$, p. 66 .

Traduction, unité de, $\mathrm{n}^{\circ} 2$, p. 144.

Transcodage, $\mathrm{n}^{\circ} 1$, p. 5 .
Translème, $\mathrm{n}^{\circ} 3$, p. 258.

Transphrastique, no 3, p. 263.

Transposition, $\mathrm{n}^{\circ} 2$, p. 151

Transtextuelle, no 3, p. 263.

Travailleur marginal, no 3 , p. 306

Triène, no 4 , p. 389.

Trois niveaux de la recherche terminologique, $n^{\circ} 3$, p. 286.

Trouble de l'encodage, $n^{\circ} 1, p .107$

Trouble de la contiguité, no 1, p. 107

Trouble de la similarité, no 1, p. 107.

Trouble du décodage, $\mathrm{n}^{\circ} 1$, p. 107.

Troubles arthriques, $n^{\circ} 1$, p. 107.

Type d'interrogations, no 4 , p. 371 .

Typologie des fautes, $n^{\circ} 2$, p. 146

Typologie des notes, $n^{\circ} 2$, p. 145 .

Unilingue, $n^{\circ} 1$, p. 20.

Unité de traduction, n० 2, p. 144.

Universaux phonétiques, $n^{\circ} 1$, p. 46 .

Universaux sémantiques, $n^{\circ} 1$, p. 46 .

Usage, $n^{\circ} 2$, p. 175

Utilité marginale, no 3 , p. 306

Verbaliser, $n^{\circ} 2$, p. 181

Vérité et mensonge, no ${ }^{\circ}$, p. 136.

Vidéotex diffusé, no 2, p. 195.

Vidéotex interactif, no 2 , p. 195.

Visioconférence, $n^{\circ} 2$, p. 195.

Visiophone, $n^{\circ} 2$, p. 195

Volume spécifique, no 2 , p. 197.

Vouloir dire, $n^{\circ} 2$, p. 154.

Wagon d'extinction, no 3, p. 282.

Wernicke, aire de, $\mathrm{n}^{\circ} 1, \mathrm{p} .13$.

Wernicke, aphasie de, $\mathrm{n}^{\circ} 1$, p. 14

Zag, no 2, p. 184

Zone cérébrale, no 1 , p. 6

Zone de Heschl, no 1, p. 13.

Zone du langage, $n^{\circ} 1$, p. $10 ; n^{\circ} 1$, p. 11 


\section{Index anglais des mots et des sujets traités}

A Linguistic Theory of Translation, $\mathrm{n}^{\circ} 2$, p. 152.

Absorptivity, $\mathrm{n}^{\circ} 2$, p. 198.

Accounting policies, $\mathrm{n}^{\circ} 2$, p. 149

Accounting record, $n^{\circ} 2$, p. 148.

Acyclic hydrocarbon, $n^{\circ} 4$, p. 388.

Add-on telephone accessories, ${ }^{\circ} 2$ 2, p. 194.

Adjacency strategy, $\mathrm{n}^{\circ} 1$, p. 87.

After Babel, $\mathrm{n}^{\circ} 2$, p. $116 ; \mathrm{n}^{\circ} 2$, p. 156 .

Agrammatic aphasia, $n^{\circ} 1$, p. 106

Agrammatisme, no 1, p. 106.

Air heater, $n^{\circ} 2$, p. 198

Alexia, no 1, p. 106

Alexia with agraphia, $n^{0} 1$, p. 106

Alice in Wonderland, $n^{\circ} 3$, p. 266

Alicyclic hydrocarbon, no 4, p. 391.

Alkadiene, $n^{\circ} 4$, p. 389.

Alkadiyne, $n^{\circ} 4$, p. 390

Alkane, $n^{\circ} 4$, p. 388 .

Alkatriene, no 4 , p. 389

Alkene, no 4, p. 389.

Alkenyl, no 4, p. 389.

Alkyl, no 4, p. 389.

Alkyne, $n^{\circ} 4$, p. 390

Amnes(t)ic aphasia, no 1, p. 106.

Anaphora, $\mathrm{n}^{\circ} 2$, p. 129.

Anarthria, no 1, p. 106

Anchor pattern, no 3, p. 316.

Anisomorphism, no 3, p. 235.

Anomia; $n^{\circ} 1$, p. $106 ; n^{\circ} 1$, p. 107

Anomic aphasia, $n^{\circ} 1$, p. 106.

Answering and recording unit, $n^{\circ} 2$, p. 195.

Answering unit, $\mathrm{n}^{\circ} 2$, p. 195.

Antireflective coating, $\mathrm{n}^{\circ} 2$, p. 199.

Aphasia, $n^{\circ} 1$, p. 106 .

Aphasia from a lesion in the region of the angular gyrus, $\mathrm{n}^{\circ} 1, \mathrm{p} .107$.

Aphemia, no 1, p. 107.

Apractic aphasia, $\mathrm{n}^{\mathrm{0}}$ 1, p. 106.

Apraxia, $\mathrm{n}^{\circ}$ 1, p. 107.

Apraxia of speech, no 1 , p. 107

Arene, $n^{\circ} 4$, p. 391.

Aromatic carbon, $\mathrm{n}^{\circ} 4$, p. 391.

Aromatic hydrocarbon, no 4, p. 391

Articulatory apraxia, $\mathrm{n}^{\circ} 1, \mathrm{p} .107$.

Articulatory impairment, $\mathrm{n}^{\circ} 1$, p. 107.

Artificial person, $\mathbf{n}^{\circ} 3$, p. 305 .

Audioconferencing, $\mathrm{n}^{\circ} 2$, p. 194.

Audiographic teleconferencing, $n^{\circ} 2$, p. 195

Audit, no 2, p. 149.

Auditor, $\mathrm{n}^{\circ} 2$, p. 149.
Authentic translation, $\mathrm{n}^{\circ} 2$, p. 128.

Autodialer, no 2, p. 194.

Automatic dialer, $n^{\circ} 2$, p. 194

Back saw, no 3, p. 291.

Backed saw, no 3, p. 290

Beginning, sense of, $\mathrm{n}^{\circ} 2$, p. 115.

Benzene ring, $n^{\circ} 4$, p. 391.

Blast primer, $n^{\circ} 3$, p. 317.

Body corporate, $\mathrm{n}^{\mathrm{O}} 3$, p. 305

Borrow, $\mathrm{n}^{\circ} 2$, p. 154.

Bracketing strategy, no 1, p. 83.

Bridged hydrocarbon, no 4, p. 393.

Broad open pit saw, no 2 , p. 183.

Broadcast videotex, $\mathrm{n}^{\circ} 2$, p. 195.

Broca's aphasia, $\mathrm{n}^{\circ} 1$, p. 106

Broken space, $\mathrm{n}^{\circ} 2$, p. 185.

Brushability, $\mathrm{n}^{\circ} 3$, p. 281.

Bus network, n० 2, p. 195

Business corporation, no 3 , p. 298 ; no 3, p. 305.

Calcareous deposit, na 3 , p. 316

Calcareous scale, $\mathrm{n}^{\circ} 3$, p. 316

Call director, $\mathrm{n}^{\circ} 2$, p. 195.

Call forwarding, $\mathrm{n}^{\circ} 2$, p. 195.

Callback, n $^{\circ} 2$, p. 195.

Caprock, no 2, p. 197.

Case gap, no 3 , p. 236.

Case parameter, no 3, p. 242.

Cataphora, $\mathrm{n}^{\circ} 2$, p. 130.

Cataphoric pronominalization, $n^{0} 2$, p. 130

Catford, J.C., no 2, p. 152.

Chairperson, $\mathrm{n}^{\circ} 2$, p. 219.

Chemurgy, no 3 , p. 282.

Chest saw, n० 2, p. 185.

Circuit switching, no 2, p. 194.

Closely held corporation, $n^{\circ} 3$, p. 305.

Closure, no 1 , p. 83

Closure strategy, $\mathrm{n}^{\circ} 1$, p. 83.

Coal tar enamel, $n^{\circ} 3$, p. 316.

Coaxial cable, $\mathrm{n}^{\circ} 2$, p. 194.

Code-switching, $\mathrm{n}^{\circ} 2$, p. 157.

Cognitive experience, $n^{\circ} 2$, p. 153

Cognitive strategies, $n^{\circ} 1$, p. 81 .

Cognitive value, $\mathrm{n}^{\circ} 2$, p. 154 .

Cohesion device, no 2 , p. 129.

Coke car, no 3 , p. 281.

Collocational shock, $n^{\circ} 2$, p. 156.

Comparative approach, $\mathrm{n}^{\circ} 2$, p. 152.

Compass saw, $n^{\circ} 2$, p. $182 ; n^{\circ} 2$, p. $185 ; n^{\circ} 2$, p. 187.

Computer teleconferencing, $n^{\circ} 2$, p. $194 ; n^{\circ} 2$, p. 195.

Concentrator, $n^{\circ} 2$, p. 198.

Concentrator collector, $n^{\circ} 2$, p. 199

Concentrting, $\mathrm{n}^{\circ} 2$, p. 198

Conceptual problem, $n^{\circ} 2$, p. 118.

Concrete sphere, $n^{\circ} 2$, p. 118.

Condensed ring, $n^{\circ} 4$, p. 392.

Conduction aphasia, $\mathrm{n}^{\mathrm{o}} 1, \mathrm{p} .106$.

Conjunction, no 2 , p. $128 ; n^{\circ} 2$, p. 131

Console, $n^{\circ} 2$, p. 195 
Constituent, $n^{\circ} 2$, p. 117.

Conte traditionnel, $\mathrm{n}^{\circ} 2$, p. 141

Context, $\mathrm{n}^{\circ} 2$, p. 155.

Contextual correlative, $n^{\circ} 2$, p. 120

Contextuality, first degree of, $n^{\circ} 2$, p. 124

Contiguity disorder, $\mathrm{n}^{\circ} 1$, p. 107.

Control, no 2, p. 147.

Corrosion preventing oil, no 3 , p. 316.

Cortical dysarthria, no 1, p. 107.

Cortical motor aphasia, no 1 , p. 106.

Cortical sensory aphasia, no 1, p. 107.

Cross-cut hand saw, no 2 , p. 185 .

Cross-cut saw, no 2 , p. $183 ; n^{\circ} 2$, p. 185

Cross-cutting saw, $\mathrm{n}^{\circ} 2$, p. 183 .

Crosscut handsaw, no 2 , p. 185

Cultural distance, $n^{\circ} 2$, p. 119.

Cultural representamen, $n^{\circ} 2$, p. 121

Cultural shock, $\mathrm{n}^{\circ} 2$, p. 156.

Cyclane, no 4 , p. 391.

Cyclene, $n^{\circ} 4$, p. 391.

Cyclo-olefin, no 4 , p. 391

Cycloalkane, $n^{\circ} 4$, p. 391

Cycloalkene, no 4, p. 391.

Cycloalkyne, $n^{\circ} 4$, p. 391

Cycloparaffin, $n^{\circ} 4$, p. 391.

Decoding, $n^{\circ} 2$, p. $120 ; n^{\circ} 3$, p. 241.

Decoding disorder, $\mathrm{n}^{\circ} 1$, p. 107.

Deferral, no 2 , p. 122.

Definitivization, $\mathrm{n}^{\circ} 2$, p. 130

Degree of redundancy, $\mathrm{n}^{\mathrm{O}} 3$, p. 230.

Derivational morphology, $\mathrm{n}^{\circ} 2$, p. 130

Developmental dysgraphia, $\mathrm{n}^{\circ} 1$, p. 107.

Developmental dyslexia, $n^{\circ} 1$, p. 107

Developmental dysphasia, $n^{\circ} 1$, p. 107

Diene, $n^{\circ} 4$, p. 389.

Digital technique, $\mathrm{n}^{\circ} 2$, p. 195

Diolefin, no 4, p. 389 .

Discursive level, $n^{\circ} 2$, p. 123.

Dog leg, $n^{\circ} 3$, p. 281.

Drogman, no 2, p. 115.

DTMF pad, n' 2, p. 194

DTMF set, no 2, p. 195.

Dual-tome multifrequency set, $\mathrm{n}^{\circ} 2$, p. 195 .

Dual-tone multifrequency pad, ${ }^{\circ} 2$, p. 194.

Dubbing, $\mathrm{n}^{\circ} 2$, p. 217.

Dynamic state, $\mathrm{n}^{\circ} 2$, p. 119.

Dyspraxia, no 1 , p. 107.

Dysprosody, no 1 , p. 107.

Efferent motor aphasia, $\mathfrak{n}^{\circ}$ 1, p. 106.

Electronic (control) unit, $\mathrm{n}^{\circ} 2$, p. 194

Electronic mail, no 2, p. 194

Electronic mailbox, $n^{\circ} 2$, p. 194.

Electronic switching, $\mathrm{n}^{\circ} 2$, p. 194.

Ellipsis, $n^{\circ} 2$, p. 128.

Elliptical formulation, $n^{\circ} 3$, p. 236

Emissivity, no 2, p. 199

Encoding, $0^{\circ} 2$, p. 120.

Encoding disorder, $\mathrm{n}^{\circ}$ 1, p. 107.

Encyclopedic knowledge, no 2, p. 156

Epistemology in translation, $n^{\circ} 2$, p. 118 .
Eutectic mixture, no 2, p. 199.

Eutectic salt, $n^{\circ} 2$, p. 199.

Evaluation, no 2, p. 133

Explicitation, no 3 , p. 236

Expressive aphasia, no 1, p. 106.

Extra-linguistic background, no 3, p. 239.

Extra-textual information, no 3 , p. 237.

Extralinguistic referent, $n^{\circ} 3$, p. 229.

Facsimile (fax), no 2, p. 195.

Facsimile device, no 2, p. 195.

Felloe saw, no 2, p. 183

Financial statement, $n^{\circ} 2$, p. 148

Fine hand saw, no 2, p. 185.

Fine panel, $n^{\circ} 2$, p. 185 .

First degree of contextuality, $n^{\circ} 2, p .124$

Fixed marginal cost, n० 3, p. 308.

Flooring saw, $\mathrm{n}^{\circ} 2$, p. $182 ; \mathrm{n}^{\circ} 2$, p. 188.

Focusing, no 2, p. 198.

Fonagy, Ivan, $n^{\circ} 2$, p. 124.

Free translation, $\mathrm{n}^{\circ} 2, \mathrm{p} .116$.

Fret saw, no 2, p. 187.

Frontal aphasia, no 1 , p. 106

Full Broca's aphasia, no 1, p. 107.

Full Wernicke's aphasia, no 1, p. 107.

Function, interrelationship of, $\mathbf{n}^{\circ} 2$, p. 117.

Fused polycyclic hydrocarbon, no 4 , p. 392

Fused ring, no 4, p. 392.

Gadamer, no 2, p. 117.

Gardner's saw, no 2, p. 187.

Gateway, no 2, p. 194

General partner, no 3 , p. 298

Gentleman's saw, $n^{\circ} 2$, p. 187

Global aphasia, no 1 , p. 106.

Glumberman's saw, $n^{\circ} 2$, p. 183 .

Grafter, $n^{\circ} 2$, p. 186

Grafter saw, $\mathrm{n}^{\circ} 2$, p. 186

Grafting saw, $n^{\circ} 2$, p. $182 ; n^{\circ} 2$, p. $185 ; n^{\circ} 2$, p. 180 .

Graphic processor, no 2, p. 195.

Gravel-bed heat, no 2, p. 198

Green rot, $n^{\circ} 3$, p. 316

Greenhouse effect, no 2, p. 199

Half rip saw, $n^{\circ} 2$, p. 185 .

Hand (crosscut), n ${ }^{\circ} 2$, p. 185.

Hand rip saw, no 2, p. 186.

Hand saw, no 2 , p. $182 ; n^{\circ} 2$, p. $184 ; n^{\circ} 2$, p. 185

Handled saw, $\mathrm{n}^{\circ} 2$, p. 182

Handsaw, no 2 , p. 184

Handsfree unit, $n^{\circ} 2$, p. 194.

Harris, $n^{\circ} 2$, p. 118

Heliotechnology, no 2, p. 199.

Hermeneutic Motion, no 2, p. 156.

Hermeneutic process, $n^{\circ} 2$, p. 125

History of translation, $\mathrm{n}^{0} 2$, p. 115

Hjelmslev, $n^{\circ} 2$, p. 122.

Homologous serie, $n^{\circ} 4$, p. 388.

Homologue, $n^{\circ} 4$, p. 388 
Hothouse effect, $\mathrm{n}^{\circ} 2$, p. 199.

Hybrid term, $\mathrm{n}^{\circ} 2$, p. 116.

Hydrocarbon, no 4, p. 387.

Iconographical equivalent, $n^{\circ} 3$, p. 239.

Idiomatic expression, $\mathrm{n}^{\circ} 2$, p. 152.

Implicitation, $\mathrm{n}^{\circ} 3$, p. 242.

Impressive aphasia, no 1, p. 106.

Inflectional morphology, no 2 , p. 130

Information provider (IP), $\mathrm{n}^{\circ} 2$, p. 194.

Inquiry, $\mathrm{n}^{\circ} 2$, p. 152.

Inside start saw, no 2, p. 188.

Integrated services digital network (ISDN), $\mathrm{n}^{\circ} 2$, p. 195.

Intelligent terminal, $\mathrm{n}^{\circ} 2$, p. 195.

Interactive videotex, $\mathrm{n}^{\circ} 2$, p. 195

Internal control, $\mathrm{n}^{\circ} 2$, p. 148.

Interpretant, $\mathrm{n}^{\circ} 2$, p. 117.

Interrelationship of function, $\mathrm{n}^{\circ} 2, \mathrm{p} .117$.

Interseasonal storage, $n^{\circ} 2$, p. 199.

Intersubjective evaluation, $\mathrm{n}^{\circ} 2$, p. 119.

Intersubjective process, $\mathrm{n}^{\circ} 2$, p. 120 .

Intranlingual communication, $\mathrm{n}^{\circ} 2$, p. 154

Invariant core, $\mathrm{n}^{\mathrm{0}} 2$, p. $124 ; \mathrm{n}^{\mathrm{0}} 2$, p. 125.

Isochart, $n^{\circ} 3$, p. 317.

Isocorrosion chart, $n^{\circ} 3$, p. 317

Isocorrosium diagram, $n^{\circ} 3$, p. 317

Isolation (of the speech area) syndrome, $n^{\circ} 1$, p. 107.

Isomorphic relationship, $\mathrm{n}^{\circ} 2$, p. 116

Isomorphic topology, no 2 , p. 125.

Jakobson, no 2 , p. 154.

Johansen, $n^{\circ} 2$, p. 123 .

Johansen, Jorgen Dines, n० 2, p. 121.

Joint stock company, no 3 , p. 298.

Key, no 2, p. 195.

Keyhole saw, no 2 , p. $182 ; n^{\circ} 2$, p. $185 ; n^{\circ} 2$, p. 187.

Kinetic motor aphasia, no 1 , p. 106.

Language processing, $n^{\circ} 1$, p. 81 .

Language typonymy, no 2 , p. 157.

Language varieties, $n^{\circ} 2$, p. 155.

Law of eventually diminishing marginal physical productivity, no 3 , p. 308 .

Legalese, $n^{\circ} 2$, p. 155.

Levy, Jiri, no 2, p. 125.

Lexical cohesion, no 2 , p. 129.

Lexical repetition, $\mathrm{n}^{\circ} 2$, p. 130 .

Lexicography in the Electronic Age, no 4, p. 415.

Limited partner, no 3, p. 298.

Limited partnership, n० 3 , p. 298.

Linguistic cohesion, $\mathrm{n}^{\circ} 2$, p. 128.

Linguistic presupposition, no 2 , p. 128.

Linguistic untranslatability, no 2, p. 156.

Literal translation, $\mathrm{n}^{\circ} 2$, p. 152.
Literariness, $n^{\circ} 2$, p. 124.

Loan blend, no 3 , p. 280.

Loan-object, n० 2 , p. 156

Loan-shift, n० 3, p. 280.

Loan translation, $n^{\circ} 3$, p. 281.

Loan-word, no 2 , p. $156 ; n^{\circ} 3$, p. 280.

Local area network (LAN), no 2, p. 195.

Localizer, no 3 , p. 282.

Lock saw, no 2, p. 187.

Lockup converter, $n^{\circ} 3$, p. 233.

Long saw, $n^{\circ} 2$, p. 183.

Loudspeaker, no2, p. 194.

Management, $n^{\circ} 2$, p. 147.

Marginal analysis, $n^{\circ} 3$, p. 308 .

Marginal condition, no 3 , p. 309.

Marginal equality, n०3, p. 309.

Meaning, transference of, $n^{\circ} 2$, p. 153.

Megatype, $n^{\circ} 2$, p. 125 .

Message, no 2, p. 153.

Messaging software, no 2, p. 194.

Metalinguistic focus, $n^{\circ} 3$, p. 242.

Métaphore en phonétique, n०2, p. 124.

Metatext, $n^{\circ} 2$, p. 124.

Minimax strategy, no2, p. 125

Motor aphasia, no 1, p. 106.

Mounin, Georges, n० 2, p. 156 .

Mukarovsky, no2, p. 119.

Multiple addressing, no2, p. 194.

Narrow open pit saw, n०2, p. 183.

Nomenclature, $n^{\circ} 3$, p. 230.

Nominalisation, $n^{\circ} 3$, p. 236.

Non-ambiguity strategy, $\mathrm{n}^{\circ} 1$, p. 83.

Nonfluent aphasia, no 1 , p. 106.

Nonspecific Language Practice texts, n०2, p. 122.

Normal form, $\mathrm{n}^{\circ} 1$, p. 83

Normal form strategy, no 1, p. 83.

Object, no 2, p. 117

Olefin, no 4 , p. 389.

On Poetic Language, n०2, p. 119.

One-man cross cut saw, no 2, p. 184.

One-man crosscut saw, n०2, p. $182 ; n^{\circ} 2$, p. 184

One-to-one correspondance, no 3 , p. 230.

One-to-one relationship, no 2, p. 154 .

Open pit saw, n०2, p. 183.

Open pit saw (broad), no 2, p. 182.

Open pit saw (narrow), no 2, p. 182

Operation, n' 2, p. 147.

Optical fiber, no 2, p. 194.

Optimal solution, $\mathrm{n}^{\circ} 2$, p. 125.

Organic chemistry, no 4 , p. 387 .

Organization, $n^{\circ} 2$, p. 147 .

Overburden, $n^{\circ} 2$, p. 197.

Oxygen scavenger, no 3 , p. 317. 
Pack shot, no 3, p. 282.

Packet switching, no 2, p. 194.

Pad-saw, $n^{\circ} 2$, p. 187

Panel saw, $n^{\circ} 2$, p. $182 ; n^{\circ} 2$, p. $185 ; n^{\circ} 2$, p. 187.

Pannel saw, no 2, p. 187.

Para-textual system, no 3 , p. 240.

Paraffin, $n^{\circ} 4$, p. 388.

Paragrammatism, $\mathrm{n}^{\circ} 1$, p. 107.

Paragraphia, no 1 , p. 107

Paralexia, no 1, p. 107.

Parallel function, $n^{\circ} 1$, p. 87.

Paraphasia, no 1, p. 107.

Paraphrase, $\mathrm{n}^{\circ} 2$, p. 116.

Parieto-angular gyrus syndrome, $\mathrm{n}^{\circ} \mathrm{1}, \mathrm{p} .107$.

Partial equivalence, $n^{\circ} 2$, p. 116

Pattermaker's saw, no 2, p. 185.

Pebble-bed, n० 2, p. 198.

Peirce, $\mathrm{n}^{\circ} 2$, p. 117

Peripheral motor aphasia, $\mathrm{n}^{\circ} 1$, p. 106.

Photothermal conversion, $\mathrm{n}^{\mathrm{0}} 2$, p. 199.

Phonematic realization aphasia, $\mathrm{n}^{\circ} 1, \mathrm{p} .106$.

Phonetic disintegration, $n^{\circ} 1$, p. 107.

Phonetic disintegration syndrome, no 1, p. 107.

Photo timer, no 3, p. 282.

Pit saw, $n^{\circ} 2$, p. 183.

Pit turning saw, n०2, p. 183 .

Plumber's saw, no 2, p. 185.

Poetic text, no 3 , p. 228.

Policy, no 2, p. 149.

Polyene, $n^{\circ} 4$, p. 389

Polysemy, no 2, p. 156.

Popovic, Anton, $\mathrm{n}^{\circ} 2$, p. 122.

Popper, Karl, no 2, p. 126.

Port saw, no 2 , p. 187.

Practice of technical translation, no 3, p. 228.

Pragmatic aphasia, no 1, p. 107.

Pragmatic cohesion, no 2, p. 128.

Pragmatic dimension, $n^{\circ}$ 2, p. 125.

Pragmatic model, no 2, p. 121.

Pragmatic presupposition, $\mathrm{n}^{\circ} 2$, p. 128.

Pragmatics of translation, $n^{\circ} 2$, p. 115.

Preconstruction primer, $n^{\circ} 3$, p. 317.

Prefabrication primer, $n^{\circ} 3$, p. 317.

Principle of sameness, $n^{\circ} 2$, p. 118 .

Private branch exchange (PBX), no 2 , p. 194

Private company, $n^{\circ} 3$, p. $299 ; n^{\circ} 3$, p. 300.

Private sector, $n^{\circ} 3$, p. 300 .

Process, $n^{\circ} 2$, p. 118

Profile, no 3, p. 316

Pronominalization, $\mathrm{n}^{\circ} 2$, p. 130

Pruning saw, $n^{\circ} 2$, p. $182 ; n^{\circ} 2$, p. $185 ; n^{\circ} 2$, p. 187.

Public company, no 3, p. 299.

Public sector, $n^{\circ} 3$, p. 300.

Pulse signalling keypad, n०2, p. 194.

Pure anarthria, no 1, p. 106.

Pure motor aphasia, $\mathrm{n}^{\circ} 1$, p. 106.

Pure word dumbness, no 1 , p. 107.
Qua text, $n^{\circ} 3$, p. 229.

Radiation coating, $\mathrm{n}^{\circ} 2$, p. 199.

Rank-bound, no 2, p. 152

Receptive aphasia, $\mathrm{n}^{\circ} 1, \mathrm{p} .106$.

Recessive trait, $n^{\circ}$ 2, p. 123

Reference, $n^{\circ} 2$, p. 128.

Referential function, $\mathrm{n}^{\circ} 3$, p. 228

Referential input, $n^{\circ} 3$, p. 242.

Referentiality, $n^{\circ} 2$, p. 118 .

Relabelling, $\mathrm{n}^{\circ} 3$, p. 232

Remote control answering unit, $\mathrm{n}^{\mathrm{0}} 2$, p. 195.

Representamen, no 2, p. 122.

Residual disparity, $\mathrm{n}^{\circ} 2$, p. 118.

Responsibility of management, $n^{\circ} 2$, p. 150.

Restricted translation, $\mathrm{n}^{\circ} 2$, p. 152.

Ring assembly, $n^{0} 4$, p. 392.

Ring network, $\mathrm{n}^{\circ} 2, \mathrm{p} .195$.

Rip saw, n०2, p. $182 ; n^{\circ} 2$, p. $185 ; n^{\circ} 2$, p. 186.

Ripping saw, $n^{\circ} 2$, p. 186.

Ripsaw, n² 2, p. 186.

Rock (bed), no 2, p. 198

Rock-pile, no 2, p. 198

Rotary dial telephone set, no 2, p. 195

Sameness, principle of, $n^{\circ} 2$, p. 118.

Saturated, $n^{\circ} 4$, p. 387.

Saw, back, no 3, p. 291.

Saw, cranked, no 3, p. 293.

Saw, dovetail, no 3 , p. 291

Saw, dowel, no 3, p. 293

Saw, miter-box, no 3, p. 293.

Saw, mitre-box, no 3, p. 293.

Saw, pin, $n^{\circ} 3$, p. 293.

Saw, stair, no 3, p. 294.

Saw, veneer, n 3, p. 294.

Scientific text, $n^{\circ} 2$, p. 123 .

Seal, no 3, p. 317

Sealant, $n^{\circ} 3$, p. 317

Sealer, $n^{\circ} 3$, p. 317.

Sealing coat, $n^{\circ} 3$, p. 317 .

Selective surface, no 2 , p. 199

Semantic Dynamics, no 2, p. 119.

Semiosis, $\mathrm{n}^{0} 2$, p. 121.

Semiotic structure, no 3 , p. 228.

Semiotic topology, $n^{\circ} 2$, p. 119.

Sense for sense translation, $\mathrm{n}^{\circ} 2$, p. 116

Sense of a beginning, $n^{\circ} 2$, p. 115 .

Sensory aphasia, $n^{\circ} 1$, p. 107

Shared exponence, $\mathrm{n}^{\circ} 2$, p. 156

Shop primer, $n^{\circ} 3$, p. 317 .

Siding saw, no 2 , p. 185

Siemens, $n^{\circ} 4$, p. 359 .

Sign, $n^{\circ} 2$, p. $117 ; n^{\circ} 2$, p. 118 .

Sign Concepts, Semiosis and Meaning, no 2, p. 121

Sign, specificity of, $\mathrm{n}^{\mathrm{o}} 2, \mathrm{p} .121$.

Silent block, no 3, p. 282.

Siliconising, $n^{\circ} 3$, p. 317 .

Siliconizing, $\mathrm{n}^{\circ} 3$, p. 317 .

Similarity disorder, $\mathrm{n}^{\circ} 1$, p. 107.

Situation, $\mathrm{n}^{\circ} 2$, p. 155.

Situation analysis, $n^{\circ} 3$, p. 238 
Solar collector, no 2, p. 199

Solar engineering, $\mathrm{n}^{\circ}$ 2, p. 199.

Source culture, no 2, p. 126

Source text, no 3 , p. 229

Specific Language Practice texts, $n^{\circ} 2$, p. 121.

Specificity of sign, $\mathrm{n}^{\circ} 2$, p. 121.

Speech-act, $n^{\circ} 2$, p. 155.

Speed calling, $\mathrm{n}^{\circ} 2, \mathrm{p} .194$.

Spinel, no 3, p. 317

Spiro atom, no 4 , p. 394

Spiro hydrocarbon, $n^{\circ} 4$, p. 394

Spiro union, $n^{\circ} 4$, p. 394.

Star network, no 2, p. 195

Static state, $\mathrm{n}^{\mathrm{O}} 2$, p. 119

Steiner, George, $n^{\circ} 2$, p. $116 ; n^{\circ} 2$, p. 156

Stevenson, C., $\mathrm{n}^{\circ} 2$, p. 125.

Storage, $\mathrm{n}^{\circ} 2$, p. 198

Storage reservoir, no 2, p. 199

Stored program control, $\mathrm{n}^{\circ} 2$, p. 194.

Strategy, no 1 , p. 82

Stray current, $n^{\circ} 3$, p. 317

Structured lexical field, $\mathbf{n}^{\circ} 3$, p. 230.

Subcortical motor aphasia, no 1, p. 106.

Substitution, $n^{\circ} 2$, p. 128 .

Subtext, $\mathrm{n}^{\circ} 2$, p. 121

Surface profile, no 3 , p. 316

Symbolic connotation, $n^{\circ} 2$, p. 115

Symbolic syntagmata, no 3 , p. 230.

Synechdoche, no 2, p. 131.

Syntactic aphasia, no 1, p. 107.

Syntactically equivocal segment, $n^{\circ} 3$, p. 236.

Syntagmatic unit, $n^{\circ} 2$, p. 124.

System of labels, $\mathrm{n}^{0} 3$, p. 230.

Table saw, n०2, p. 185.

Target culture, no 2, p. 119 ; no 2, p. 126

Target language, n०2, p. $119 ; n^{\circ} 3$, p. 229.

Target text, n० 3 , p. 229

Taxonomy, no 2 , p. 129.

Teaching of technical translation, $n^{\circ} 3$, p. 228 ; $n^{\circ} 3$, p. 238

Technical discourse, no 3 , p. $228 ; n^{\circ} 3$, p. 238

Technical language, $n^{\circ} 3$, p. 243.

Technical register, no 3 , p. $229 ; n^{\circ} 3$, p. 243.

Technical terminology, no 3 , p. 230.

Technical translation, $n^{\circ} 3$, p. $228 ; n^{\circ} 3$, p. 229

Technical translation procedure, no 3 , p. 235

Technical translation, teaching of, no 3, p. 238.

Technical translator, no 3 , p. 243

Technical writing, no 3 , p. 243.

Teleconference room, no 2, p. 195.

Teleconferencing, $n^{\circ} 2$, p. 195

Telecopier, $n^{\circ} 2$, p. 195.

Telegraph network, n², p. 195.

Telephone answering unit, no 2, p. 195

Telephone network, no 2 , p. 195.

Teleprinter, no 2 , p. 195.

Teleterminal, no2, p. 195

Teletex, n०2, p. 195 .

Teletext, no2, p. 195 .

Teletypewriter, no 2 , p. 195.

Telewriting pad, n०2, p. 195.

Telewriting system, no 2 , p. 195.
Telex, no 2, p. 195.

Temporal aphasia, n० 1, p. 107

Temporal aphasia with sensory predominance, no 1 , p. 107.

Terminological void, no 3 , p. 234.

Text messaging, $\mathrm{n}^{\circ} 2$, p. 194

Text messaging system, n०2, p. 195.

Text-reception, n० 2, p. 117.

Textual cohesion, no 2 , p. $128 ;$ no 3 , p. 238

Textual function, $n^{\circ} 2$, p. 120 .

The Logic of Scientific Discovery, no 2, p. 126.

Theory of translation, $n^{\circ} 2$, p. $115 ; n^{\circ} 2$, p. 152 .

Thermal accumulator, no 2 , p. 198.

Thing-bound approach, n० 3 , p. 228

Thing-bound strategy, no 3 , p. 238

Three-party conference (call), no 2, p. 194

Threshold stress, no 3, p. 318 .

Thwart saw, no2, p. 183 .

Tooth, no 3 , p. 316 .

Topology, n०2, p. 116

Total translation, no 2, p. 152.

Touch dialing telephone set, n०2, p. 195.

Touch Tone telephone set, $\mathrm{n}^{\circ} 2$, p. 195.

Transcortical motor aphasia, no 1, p. 106

Transference, $n^{\circ} 2$, p. $116 ; n^{\circ} 2$, p. 154 .

Transference of meaning, $n^{\circ} 2, p .153$.

Transformation in translation, n० 2, p. 119.

Translatibility, n०2, p. 155.

Translation as Decision, $\mathrm{n}^{\circ} 2$, p. 125.

Translation equivalence, $n^{\circ} 2$, p. 155 .

Translation, history of, $n^{\circ} 2$, p. 115 .

Translation management, $n^{\circ} 4$, p. 359.

Translation, pragmatics of, $\mathrm{n}^{\circ} 2$, p. 115 .

Translation process, $n^{\circ} 3$, p. 228

Translation Propositions, no 2, p. 119

Translation, theory of, n०2, p. 115 .

Transparency, no 3 , p. 229.

Trapped water, $n^{\circ} 3$, p. 281

Triene, no 4 , p. 389 .

Tripartite sign, $n^{\circ} 2$, p. 117.

Turning saw, no 2, p. $183 ; n^{\circ} 2$, p. 187

Two-man cross cut saw, no 2, p. 183.

Two-man crosscut saw, no 2, p. 182.

Type A aphasia, no 1, p. 106.

Type I Wernicke's aphasia, no 1, p. 106

Type II Wernicke's aphasia, no 1 , p. 106.

Type III Wernicke's aphasia, no 1, p. 106

Typographical layout, no 3 , p. 240.

Underlying referent, $\mathrm{n}^{\circ} 3$, p. 239

Unincorporated business, no 3, p. 298.

Unit of translation, no 3, p. 242 .

Valence electron, no 4 , p. 387.

Verbal aphasia, no 1 , p. 107.

Verbal apraxia, no 1 , p. 107

Verstehen, no 2 , p. 117.

Videoconferencing, no 2, p. 195.

Videotelephone, $n^{\circ} 2$, p. 195.

Videotex system, n०2, p. 195

Voice mailbox, no 2 , p. 194

Voice messaging, no 2, p. 194. 
Voice messaging system, $n^{\circ} 2$, p. 195

Weathering steel, $\mathrm{n}^{\circ} 3$, p. 318 .

Wernicke's aphasia, no 1, p. 106.

Wernicke's aphasia proper, $\mathrm{n}^{\circ} 1, \mathrm{p} .106$

Wernicke's aphasia with predominant word deafness, $n^{\circ} 1$, p. 106.

Whip saw, $n^{\circ} 2$, p. 183.

Whipsaw, no 2, p. 183

Wild cat, $n^{\circ} 3$, p. 282

Wildcat, $n^{\circ} 3$, p. 282.

Wilss, Wolfram, $\mathrm{n}^{\circ} 2$, p. 121

Word-finding difficulty, $n^{\circ} 1$, p. $106 ; n^{\circ} 1$, p. 107.

'word for word' translation, $\mathrm{n}^{\mathrm{o}} 2$, p. 115.

Word muteness, no 1, p. 107.

Achevé d'imprimer à Montmagny par les travailleurs des ateliers Marquis Ltée le 6 décembre 1984. 